\title{
On the Study of Vehicle Density in Intelligent Transportation Systems
}

\author{
Julio A. Sanguesa, Fernando Naranjo, Vicente Torres-Sanz, Manuel Fogue, \\ Piedad Garrido, and Francisco J. Martinez
}

Computer Science and System Engineering Department, University of Zaragoza, C/ Atarazana 2, 44003 Teruel, Spain

Correspondence should be addressed to Francisco J. Martinez; f.martinez@unizar.es

Received 7 October 2015; Accepted 27 January 2016

Academic Editor: Manabu Tsukada

Copyright (c) 2016 Julio A. Sanguesa et al. This is an open access article distributed under the Creative Commons Attribution License, which permits unrestricted use, distribution, and reproduction in any medium, provided the original work is properly cited.

\begin{abstract}
Vehicular ad hoc networks (VANETs) are wireless communication networks which support cooperative driving among vehicles on the road. The specific characteristics of VANETs favor the development of attractive and challenging services and applications which rely on message exchanging among vehicles. These communication capabilities depend directly on the existence of nearby vehicles able to exchange information. Therefore, higher vehicle densities favor the communication among vehicles. However, vehicular communications are also strongly affected by the topology of the map (i.e., wireless signal could be attenuated due to the distance between the sender and receiver, and obstacles usually block signal transmission). In this paper, we study the influence of the roadmap topology and the number of vehicles when accounting for the vehicular communications capabilities, especially in urban scenarios. Additionally, we consider the use of two parameters: the SJ Ratio (SJR) and the Total Distance (TD), as the topology-related factors that better correlate with communications performance. Finally, we propose the use of a new density metric based on the number of vehicles, the complexity of the roadmap, and its maximum capacity. Hence, researchers will be able to accurately characterize the different urban scenarios and better validate their proposals related to cooperative Intelligent Transportation Systems based on vehicular communications.
\end{abstract}

\section{Introduction}

Modern Intelligent Transportation Systems (ITS) are being propelled by the development and adoption of wireless telecommunications and computing technologies, thereby allowing that our roads and highways can be both safer and more efficient transportation platforms.

Vehicular ad hoc networks (VANETs) are wireless communication networks which support cooperative driving among vehicles on the road. Vehicles act as communication nodes and relays, forming dynamic vehicular networks together with other nearby vehicles [1]. The specific characteristics of VANETs favor the development of attractive and challenging services and applications [2], including road safety [3], road status monitoring [4], traffic flow management [5], environmental protection [6], and mobile infotainment $[7,8]$.
In VANET-based cooperative ITS, the correct and efficient message delivery is of utmost importance. The main goal is to ensure the correct reception of messages while reducing the latency of the information received by nearby vehicles [9]. However, this dissemination of information in vehicular environments is strongly affected by (i) the signal attenuation due to the distance between the sender and receiver (especially in low vehicle density areas), (ii) the effect of obstacles in signal transmission (very usual in urban areas, e.g., due to buildings), and (iii) the instantaneous vehicle density. Regarding (i) and (ii), the topology of the roadmap is an important factor that affects the average distance between the sender and the receiver, as well as the different obstacles present in the scenario. As for (iii), the message propagation process highly depends on the number of vehicles, since lower densities can provoke message losses due to reduced communication capabilities, whereas higher densities can 
provoke reduced message delivery effectiveness due to serious redundancy, contention, and massive packet collisions caused by simultaneous forwarding, usually known as broadcast storm [10]. Although both factors (i.e., the number of vehicles and topology) play an important role in vehicular communications, we consider that they have not been well studied, especially when characterizing the different urban scenarios. In fact, we realize that VANET-aimed proposals are usually assessed under very simplistic conditions and scenarios, and we can even find that researchers surprisingly account for different concepts of vehicle density.

In this paper, we present some considerations and issues related to the number of vehicles, the roadmap topology, and its complexity, as well as the influence of these parameters in vehicular communications. Additionally, we propose the use of a new density metric which allows researchers to accurately characterize the different urban scenarios and better validate their proposals regarding cooperative Intelligent Transportation Systems. The main objective is that researchers can easily decide which scenarios to simulate when assessing their proposals.

The paper is organized as follows: in Section 2, we review some of the existing works especially related to this paper. Section 3 shows the simulation environment used to evaluate the performance of vehicular communications. In Section 4, we discuss the importance of the roadmap topology (and its complexity), as well as the number of vehicles to characterize the communication capabilities of a specific scenario. In Section 5, we present and discuss the obtained results. According to these results, in Section 6, we present a novel density metric based on the number of vehicles, the complexity of the roadmap, and its maximum capacity. Finally, Section 7 concludes this paper.

\section{Related Work}

The density of nodes is an important concept in networking, especially when accounting for communications in cooperative Intelligent Transportation Systems. In the literature, we found several works that rely on an a priori evaluation of congestion levels to improve the communication capabilities of the vehicles. Alonso and Mecklenbraeuker [11] proposed a Decentralized Congestion Control able to adapt to variable traffic densities. Das et al. [12] studied the effect of traffic density patterns on the performance of routing protocols for VANETs. Akabane et al. [13] proposed an adaptive solution for data dissemination under diverse road traffic conditions in urban scenarios. Similarly, Sanguesa et al. [14] studied the effect of very adverse vehicle density conditions on the performance of different warning message dissemination schemes.

In fact, vehicle density awareness is of crucial importance in many ITS applications, especially those related to managing emergency situations. Jin et al. [15] presented a novel warning dissemination scheme, based on the traffic flow theory, designed to mitigate the broadcast storm problem while reducing the end-to-end latency in real urban scenarios. Barrachina et al. [16] proposed four different approaches addressing the traffic congestion problem. According to them, once an emergency occurs, vehicles can perform efficient traffic redirection and emergency services can reduce their arrival time by applying evolution strategies based on the traffic density estimation obtained by means of V2I communications.

Despite the importance of vehicle density and roadmap topology in vehicular networks performance, there is no consensus when accounting for the concept of vehicle density in VANET works. Additionally, so far there have not been enough studies that explored the complexity of roadmap topology in order to improve wireless communications in vehicular environments. Next, we will discuss the most relevant works in this field.

Tyagi et al. [17] considered the problem of vehicular traffic density estimation, using the information provided by the cumulative acoustic signal acquired from a roadside-installed single microphone. This cumulative signal comprises several noise signals such as tire noise, engine noise, engine-idling noise, occasional honks, and air turbulence noise of multiple vehicles. In this work, the vehicle density is classified into three broad traffic density states, that is, jammed (where the vehicles' average speed varies from 0 to $10 \mathrm{~km} / \mathrm{h}$ ), mediumflow (10-40 km/h), and free-flow ( $40 \mathrm{~km} / \mathrm{h}$ and above).

As for the scenario used, authors collected acoustics data from the New Delhi South District area. More specifically, the data were collected from a roadside-installed omnidirectional microphone. In our opinion, authors should assess their approach in other different scenarios to better study the feasibility and accuracy of their proposal.

Tan and Chen [18] proposed a novel approach of combining an unsupervised clustering scheme called AutoClass with Hidden Markov Models (HMMs) to determine the traffic density state in a Region of Interest (ROI) of a road in a traffic video. Similarly to the previously presented work, authors considered the vehicle density in terms of four different states (i.e., empty, low, high, and full traffic density states, resp.).

Regarding the roadmap used, authors tested their algorithm on a video sequence of a traffic intersection of New South Wales. Although we consider that they should assess their proposal in other topologies, at least, they evaluated it under cloudy and sunny conditions.

Using vehicular communications makes vehicle density estimation more accurate and powerful since the messages exchanged by the vehicles in the scenario can be used to better estimate the vehicles that are present in a specific area. Maslekar et al. [19] claimed that clustering has demonstrated to be an effective concept to implement the estimation of vehicular density in the surroundings. In this work, they proposed a direction based clustering algorithm with a cluster head switching mechanism. Simulation results showed that the proposed clustering algorithm provides a better accuracy in terms of density estimation. However, due to high mobility, a stable cluster within a vehicular framework is difficult to implement. In this work, authors considered the vehicle density in terms of vehicles per square kilometer.

Regarding the topology used, authors simulated a scenario with multiple intersections spread over simulation area of $9 \mathrm{~km}^{2}$. However, authors did not provide enough 
details related to topology, especially about its complexity, and whether the topology is real or synthetic.

Venkata et al. [20] proposed a clustering approach for traffic monitoring and routing, where the cluster head $(\mathrm{CH})$ election is done based on distance and direction information. Since clusters are formed all along the road, CHs will take the responsibility of routing the message to the destination. Simulation results showed better stability, accurate density estimation in the cluster, better end-to-end delay, and good packet delivery ratio. However, the density estimation mechanism operation is limited to the vehicles within the cluster. Similarly to the previous work, authors considered the vehicle density in terms of vehicles per square kilometer.

To evaluate the performance of the proposed algorithm, authors simulated an area of $6.2 \mathrm{~km}^{2}$ with multiple intersections. However, similarly to the previous work, authors did not provide enough details to make their simulations reproducible.

In Sanguesa et al. [21], authors proposed a novel realtime mechanism to estimate the vehicular density in urban environments based on V2V communications. Their mechanism uses as input parameters the number of beacons received per vehicle and the topological characteristics of the environment where the vehicles are located. Unlike that paper, in the present work we introduce some considerations regarding how researchers determine the density of vehicles when assessing their V2V-aimed proposals. More specifically, we study in detail both the complexity of the maps and the maximum capacity of vehicles that roadmaps can host. In addition, we introduce a new metric to account for the density of vehicles. This metric, unlike the classic vehicle per $\mathrm{km}^{2}$, also refers to the capacity and complexity of maps, so it is much more recommendable to be used when evaluating new proposals related to VANETs. Using our new metric, researchers can easily determine which maps and densities to simulate when evaluating their proposals, ensuring that their conclusions will be more accurate and representative.

All these works established the importance of vehicular density awareness in vehicular environments, but as previously mentioned, it seems that there is no consensus about the concept of vehicle density and how different densities directly affect vehicular communications. Additionally, none of these works has deepened in the analysis of density, or the effect of the topology in the results obtained. In most cases, vehicle density is determined by the number of vehicles per square kilometer or simply classified into different states (e.g., empty, low, medium, and high densities).

Figure 1 presents a taxonomy of the different density metrics used in the literature, including our proposed one. We have classified existing density metrics into two different categories, that is, those metrics that measure the density in terms of traffic flow and those that consider the density in terms of vehicles located in a determined area. Unlike existing proposals, our density metric approach accounts not only for the number of vehicles but also for the capacity and complexity of the maps. In this paper, we demonstrate the influence of the topology and the number of vehicles to better characterize the vehicular scenarios, thereby predicting their communications capabilities.

\section{Simulation Environment}

To analyze and test the performance of vehicular communications we used the ns-2 simulator [22], modified to include the IEEE 802.11p [23] standard (all these improvements and modifications are available in http://www.grc.upv.es/software/). The purpose of the 802.11 p standard is to provide the minimum set of specifications required to ensure interoperability between wireless devices when attempting to communicate in potentially fast-changing communication environments. In terms of the physical layer, the data rate used for packet broadcasting is $6 \mathrm{Mbit} / \mathrm{s}$, as this is the maximum rate for broadcasting in 802.11p. The MAC layer was also extended to include four different channel access priorities. Therefore, application messages are categorized into four different Access Categories (ACs), where AC0 has the lowest and AC3 the highest priority.

The simulator was also modified to make use of our Real Attenuation and Visibility (RAV) scheme [24], which proved to increase the level of realism in VANET simulations using real urban roadmaps in the presence of obstacles. The mobility of the vehicles was generated using CityMob for Roadmaps version 2 (C4Rv2) [25], a mobility generator based on SUMO [26], able to import maps directly from OpenStreetMap (OSM) [27].

With regard to data traffic, we consider that vehicles operate in two modes: (a) warning mode and (b) normal mode. Warning mode vehicles inform other vehicles about their status by sending warning messages periodically with the highest priority at the MAC layer (similarly to the Decentralized Environmental Notification Messages (DENMs) defined by the European Telecommunications Standards Institute (ETSI) [28]); each vehicle is only allowed to propagate them once for each sequence number. Normal mode vehicles enable the diffusion of these warning packets and, periodically, they also send beacons with information such as their positions, speed, and status (similarly to the Cooperative Awareness Messages (CAMs) also defined by the ETSI). These periodic messages have lower priority than warning messages, and so they are not propagated by other vehicles.

To analyze the communications performance, we focus on the percentage of informed vehicles (i.e., the percentage of vehicles that receive the warning messages sent by warning mode vehicles) and the warning notification time, which is the time required by normal vehicles to receive a warning message sent by a warning mode vehicle. All the results included in this paper represent an average of over 50 repetitions with different random scenarios, obtaining for all of them a confidence degree of $95 \%$. Table 1 shows the main parameters used for the simulations.

\section{Roadmap Characterization in Vehicular Communications}

The roadmap (road topology) is an important factor accounting for mobility and communications in vehicular networks, since the topology constrains cars' movements, and the wireless signal can be affected by the presence of obstacles 


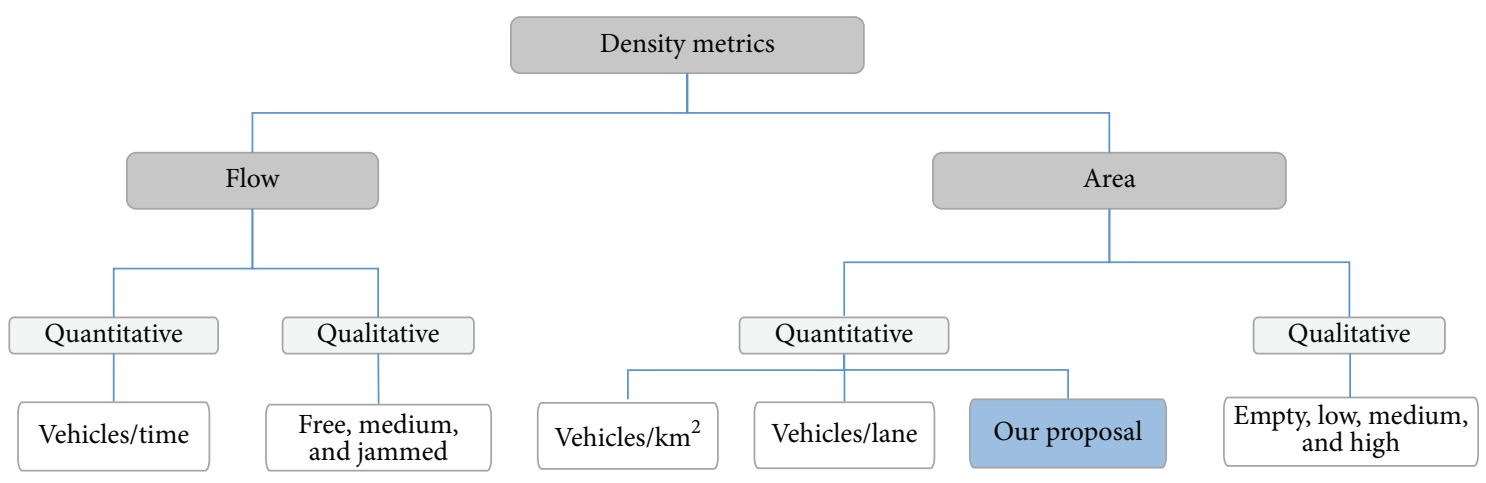

FIgURE 1: A taxonomy of vehicle density metrics.

TABLE 1: Parameter settings in the simulations.

\begin{tabular}{|c|c|}
\hline Parameter & Value \\
\hline Roadmaps & $\begin{array}{c}\text { Madrid, Beijing, Prague, Amsterdam, } \\
\text { Valencia, and San Francisco }\end{array}$ \\
\hline Number of vehicles & $100-1000$ \\
\hline Roadmap size & $2000 \mathrm{~m} \times 2000 \mathrm{~m}$ \\
\hline $\begin{array}{l}\text { Warning message } \\
\text { size }\end{array}$ & $256 \mathrm{~B}$ \\
\hline $\begin{array}{l}\text { Beacon message } \\
\text { size }\end{array}$ & $512 \mathrm{~B}$ \\
\hline $\begin{array}{l}\text { Warning messages } \\
\text { priority }\end{array}$ & AC3 \\
\hline Beacon priority & $\mathrm{ACl}$ \\
\hline $\begin{array}{l}\text { Interval between } \\
\text { messages }\end{array}$ & 1 second \\
\hline MAC/PHY & 802.11p \\
\hline $\begin{array}{l}\text { Radio propagation } \\
\text { model }\end{array}$ & RAV [24] \\
\hline Mobility model & Krauss et al. [29] \\
\hline $\begin{array}{l}\text { Channel } \\
\text { bandwidth }\end{array}$ & $6 \mathrm{Mbps}$ \\
\hline $\begin{array}{l}\text { Max. transmission } \\
\text { range }\end{array}$ & $400 \mathrm{~m}$ \\
\hline
\end{tabular}

such as buildings. Roughly described, an urban topology is a graph where vertices and edges represent, respectively, junction and road elements.

In this section, we present the different parameters that better characterize the communication capabilities of a specific roadmap in vehicular networks.

4.1. Complexity of the Map. An important issue to measure the complexity of the map is to obtain the different features of each roadmap (e.g., the number of streets, the number of junctions, the average distance of segments, and the number of lanes per street).

We consider that the parameters that better correlate with the complexity of the roadmap are the number of streets and the number of junctions [30]. Table 2 shows the values of 15 different fragments (of $4 \mathrm{~km}^{2}$ ) of real cities. We also added
TABLE 2: Number of streets, junctions, and SJR of the analyzed maps.

\begin{tabular}{lccc}
\hline & Streets & Junctions & SJ Ratio \\
\hline Houston & 548 & 650 & 0.8430 \\
Madrid & 628 & 715 & 0.8783 \\
San Francisco & 725 & 818 & 0.8863 \\
Turin & 1212 & 1353 & 0.8958 \\
Los Angeles & 287 & 306 & 0.9379 \\
Amsterdam & 1494 & 1449 & 1.0310 \\
Sydney & 872 & 814 & 1.0712 \\
Liverpool & 1758 & 1502 & 1.1704 \\
Dubai & 676 & 558 & 1.2114 \\
Brussels & 2365 & 1921 & 1.2311 \\
Prague & 3157 & 2542 & 1.2419 \\
Beijing & 777 & 618 & 1.2573 \\
Valencia & 2831 & 2233 & 1.2678 \\
Rio de Janeiro & 542 & 401 & 1.3516 \\
Rome & 1656 & 1193 & 1.3881 \\
\hline
\end{tabular}

a column labeled as SJ Ratio (SJR, which represents the result of dividing the number of streets in the number of junctions). As shown, the first 5 cities (Houston, Madrid, San Francisco, Turin, and Los Angeles) present a SJR lower than 1, which indicates that they have a simple topology, while the rest of the cities present a greater than 1 SJR value, which indicates that they have a complex topology. Also, note that although Rio de Janeiro has a relatively small number of streets and junctions, it has a complex topology. Figure 2 shows three of the topologies used in our simulations (San Francisco that represents a simple topology, Amsterdam that represents a medium complexity topology, and Valencia that represents a more complex topology).

4.1.1. The Concept of Street. As previously mentioned, to calculate the SJR it is necessary to measure the number of streets and the number of junctions. As for the number of junctions, it is only necessary to count the junctions between different street segments. However, regarding the number of streets, we realized that different alternatives could be selected to obtain the number of streets in a given roadmap. 


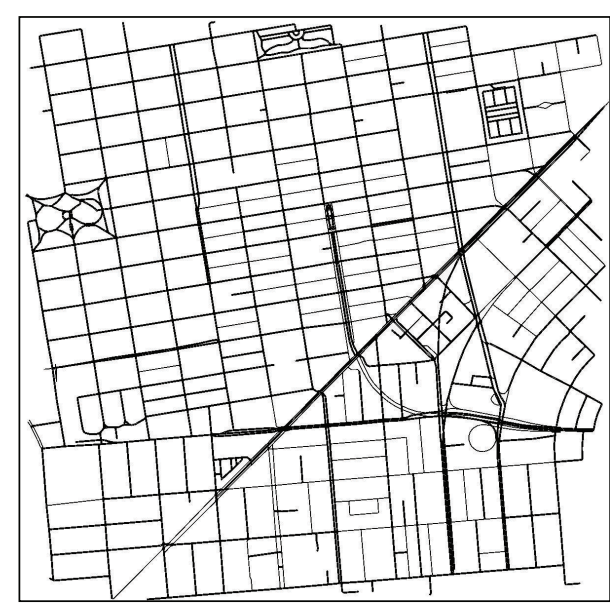

(a)

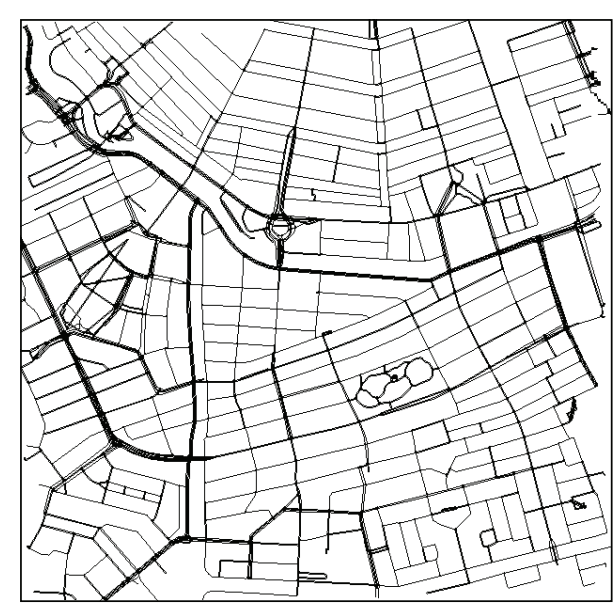

(b)

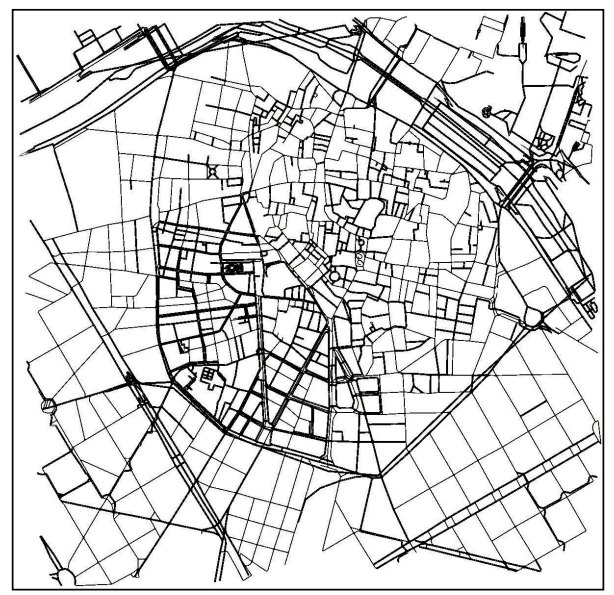

(c)

FIGURE 2: Some maps used in our simulations. Fragments of the cities of (a) San Francisco (USA), (b) Amsterdam (Netherlands), and (c) Valencia (Spain).

Basically, the different alternatives are (i) the number of streets obtained in SUMO, where each segment between two junctions is considered a street, (ii) the number of streets obtained in OpenStreetMap, where each street has a different "name," and (iii) the number of streets according to the RAV radio propagation model [24], where the visibility between vehicles is taken into consideration when identifying the different streets.

Figure 3 shows a small portion of New York City to depict the different criteria when counting the number of streets. For example, Thames street is considered only one street in OSM, whereas the SUMO and RAV models consider that there are two different streets instead. However, if we observe Cedar street, the RAV visibility model and the OSM approaches consider a single street (as expected), whereas it is represented by three different streets according to SUMO, since it has three different segments. Finally, according to both the OSM and SUMO approaches, Trinity Place and Church Street are represented as two different streets, whereas the RAV model considers only that one street exists.

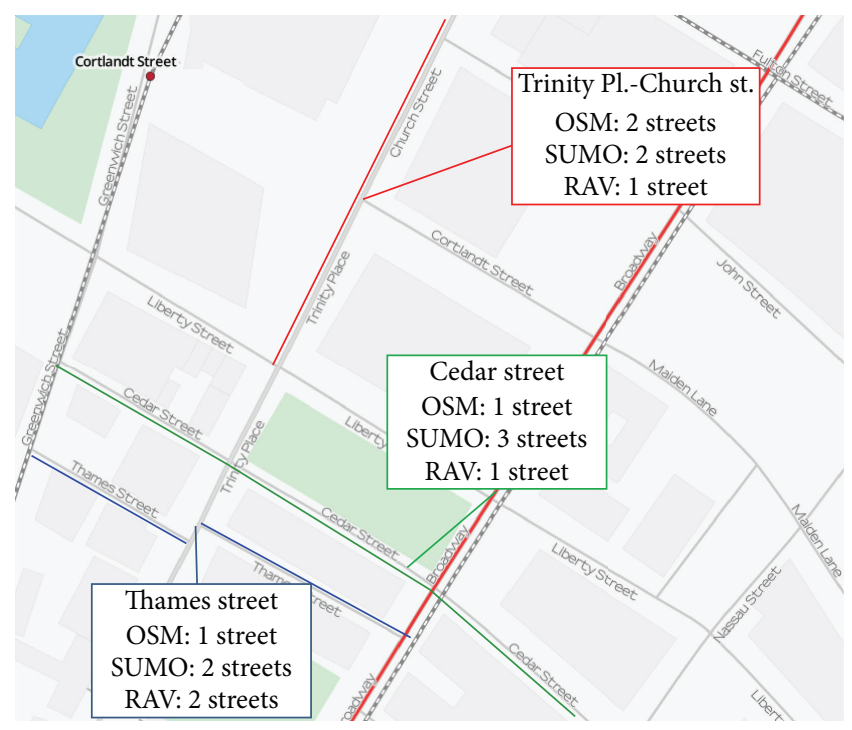

FIGURE 3: Different criteria when counting the number of streets. 
TABLE 3: Number of streets obtained depending on the criterion used.

\begin{tabular}{lccc}
\hline City & SUMO & OSM & RAV \\
\hline Rome & 2780 & 1484 & 1656 \\
Rio de Janeiro & 758 & 377 & 542 \\
Amsterdam & 3022 & 796 & 1494 \\
Madrid & 1387 & 1029 & 628 \\
\hline
\end{tabular}

Table 3 shows the values obtained when counting the number of streets of some of the cities studied, according to each criterion (i.e., SUMO, OSM, and RAV). As shown, the differences between these approaches are significant, meaning that it is important to decide which one to use in order to obtain accurate and realistic results. After some experiments [21], we realized that the third approach (i.e., RAV) better correlates with the real features of cities, since the other two present some drawbacks: they are not accurate enough, or they present some errors (e.g., SUMO always considers segments between junctions as streets, and using street names to estimate the communication between the vehicles may result in inaccurate estimations).

4.2. Size of the Map. As previously mentioned, most of researchers use the area (i.e., in terms of $\mathrm{km}^{2}$ ) to measure the size of the map. However we consider that this metric does not directly reflect the communications capabilities of a roadmap, especially in terms of vehicles informed when disseminating warning messages.

Figure 4 presents three different example scenarios with the same area. In these scenarios, we consider that vehicle $S$ sends a warning message and receivers rebroadcast this message; green vehicles do receive the message, whereas white vehicles do not receive anything either due to their distance to the sender or because of buildings blocking the wireless signal. As observed, the topology, as well as the maximum number of vehicles that could be present in the different scenarios, greatly affects the communication capabilities; they depend on the number of streets, the number of lanes of each street, and the length of them.

In particular, Figure 4 shows that the size of the map (in terms of $\mathrm{km}^{2}$ ) does not clearly affect the warning message dissemination process. In fact, the number of vehicles informed in the three examples is different, despite the fact that the number of vehicles is the same. Moreover, we can observe that the maximum number of vehicles that each example can host is also different. Hence, when focusing on the size of the map, we consider that it is also necessary to account for the number and the length of the lanes to better characterize the communication capabilities of a roadmap. However, we observe that most of researchers only account for the number of vehicles per $\mathrm{km}^{2}$ when dealing with vehicle density in vehicular environments.

A more suitable metric related to the size of the map should account for the total length of roads present in a given area, instead of the area itself, similarly to the road density metric used by the International Road Federation (IRF) in
TABLE 4: Total distance of maps analyzed.

\begin{tabular}{lc}
\hline & TD \\
\hline Rio de Janeiro & 24.8 \\
Dubai & 29.9 \\
Los Angeles & 32.3 \\
Rome & 32.8 \\
Sydney & 34.2 \\
Beijing & 35.2 \\
San Francisco & 36.3 \\
Madrid & 36.3 \\
Amsterdam & 37.1 \\
Turin & 43.0 \\
Houston & 43.6 \\
Valencia & 47.8 \\
Liverpool & 48.3 \\
Brussels & 49.3 \\
Prague & 62.1 \\
\hline
\end{tabular}

their World Road Statistics (WRS) report [31]. Nevertheless, this metric only measures the length of the roads without considering the number of lanes of each one, thus treating multilane roads and single-lane roads in the same way, which is inaccurate since the amount of vehicles able to travel in multilane roads is higher.

According to these considerations, we define the Total Distance (TD) of a map as the sum of the length of all the lanes of each street per $\mathrm{km}^{2}$

$$
\operatorname{TD}(\text { Map })=\frac{\sum_{s \in \mathbb{S}} \text { length }(s) \cdot \text { num_lanes }(s)}{\operatorname{area}_{\mathrm{km}^{2}}},
$$

where each street is denoted as $s, \mathbb{S}$ is the set of all the streets of the map, length $(s)$ is the length (in $\mathrm{km}$ ) of the street $s$, num lanes $(s)$ is the number of lanes of the street $s$, and area $_{\mathrm{km}^{2}}$ represents the area of the map in $\mathrm{km}^{2}$.

We consider that TD should be used when referring to the size of a map (indicating its maximum capacity of vehicles) when dealing with vehicular communications. Table 4 shows the information obtained after analyzing the roadmaps of different cities. As shown, the differences between the different cities are significant; some cities such as Rio de Janeiro or Dubai present TD values less than 30, whereas other cities such as Valencia, Brussels, or Liverpool present TD values close to 50. Prague presents the highest TD value by far (62.1).

4.3. Number of Vehicles in Vehicular Networks. One of the most important parameters to be considered when a message is going to be broadcasted in VANETs is the number of vehicles in a specific area. According to previous works [21, 32 ], the number of vehicles is a key factor that directly affects warning message dissemination performance in VANET scenarios. Additionally, as mentioned in Section 2, several metrics have been considered to measure density so far (such as vehicles per hour, vehicles per $\mathrm{km}^{2}$, related to the average speed of vehicles). 


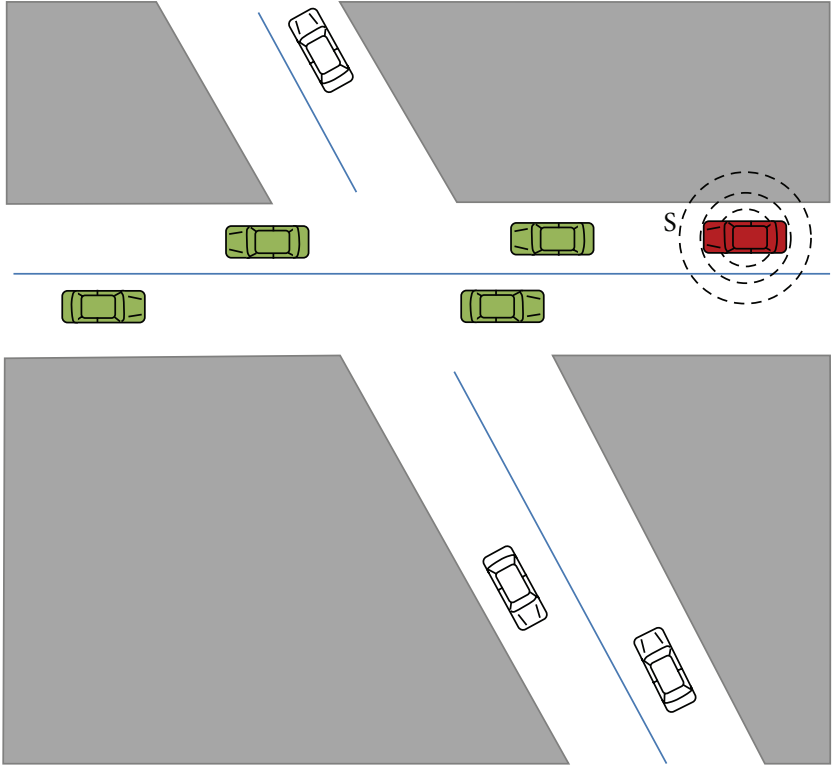

(a)
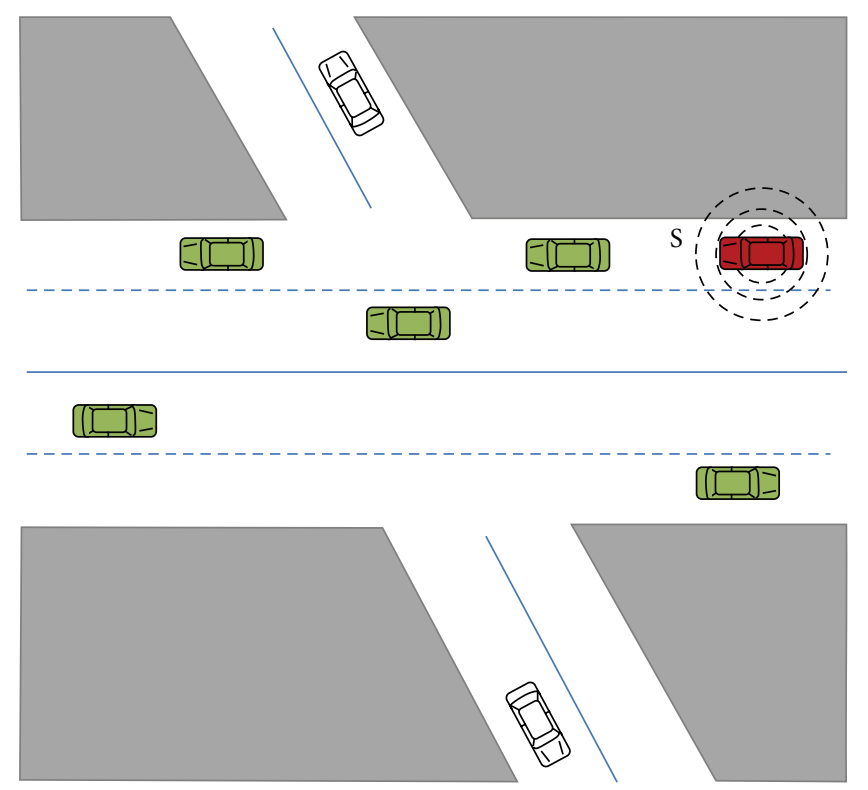

(b)

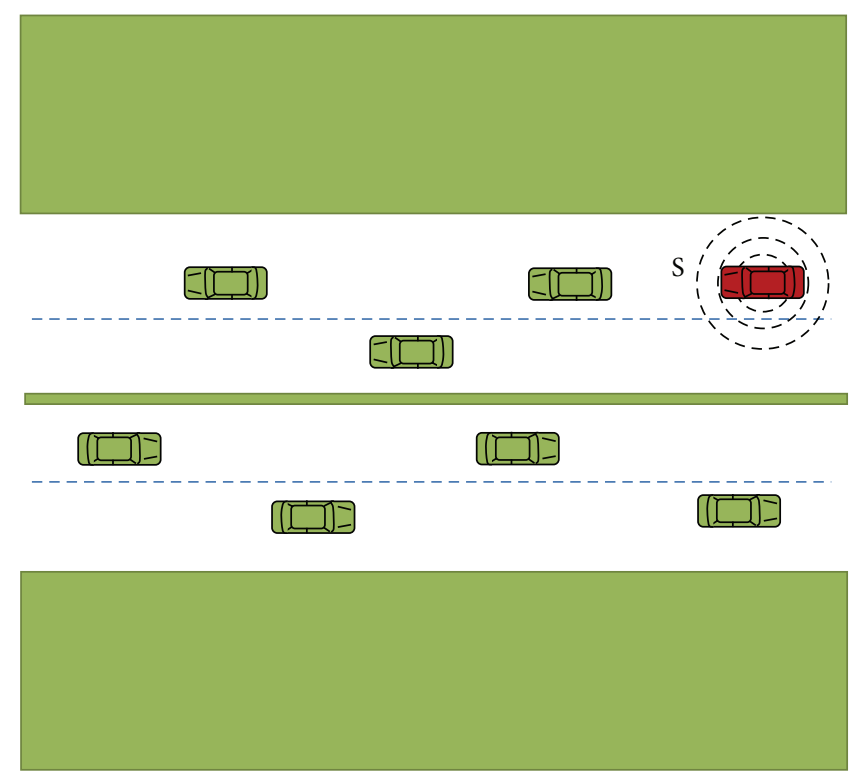

(c)

Figure 4: Map example with three different configurations: (a) single-lane, (b) multilane, and (c) highway.

Figure 5 shows the simulation results when varying the number of vehicles, in the scenarios of Valencia and San Francisco, respectively. We have tested with different numbers of vehicles, ranging from 100 to 1000 (i.e., from 25 to 250 vehicles $/ \mathrm{km}^{2}$ ). As shown, in both cases, the percentage of informed vehicles varies according to the number of vehicles. As expected, the communication possibilities (in terms of vehicles that are informed) increase when the number of vehicles also increases.

This characteristic is explained because the flooding propagation of warning messages works better with higher vehicle densities. Moreover, the topology of the map also affects the obtained results. In fact, we observe that the percentage of vehicles informed is greater in simple maps than in complex maps, where the wireless signal is usually blocked by the presence of buildings.

\section{Simulation Results: Influence of SJR and TD Parameters}

As demonstrated in the previous section, the impact of both the number of vehicles and roadmap complexity clearly affects communications performance. Moreover, we observed that researchers surprisingly consider different concepts of vehicle density. In this section, we evaluate 


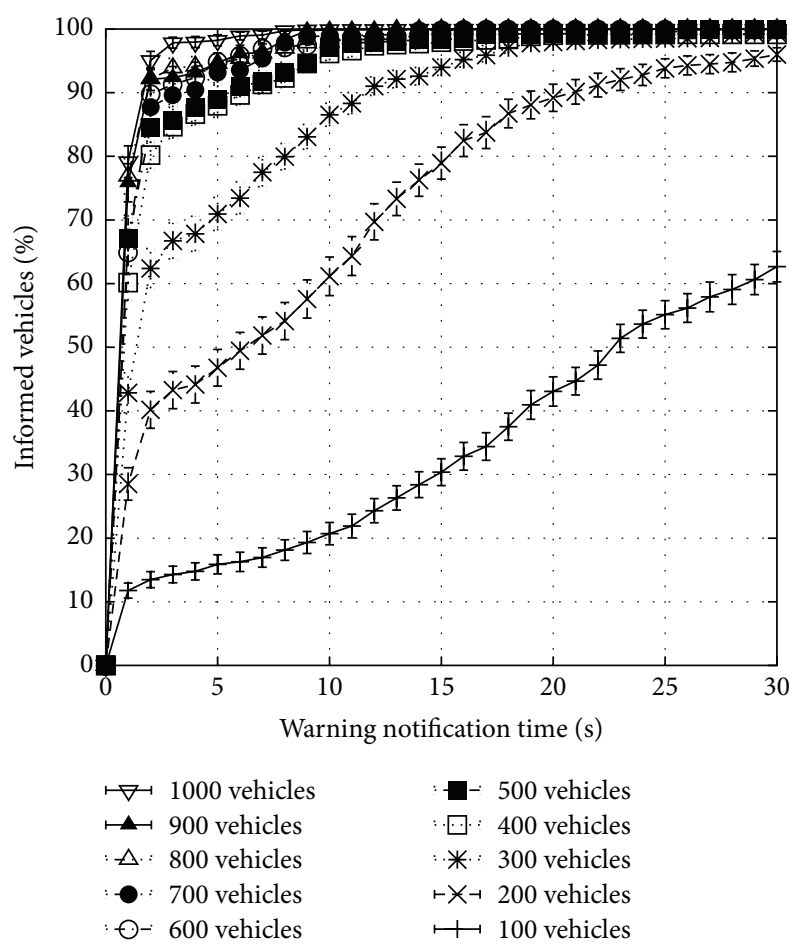

(a)

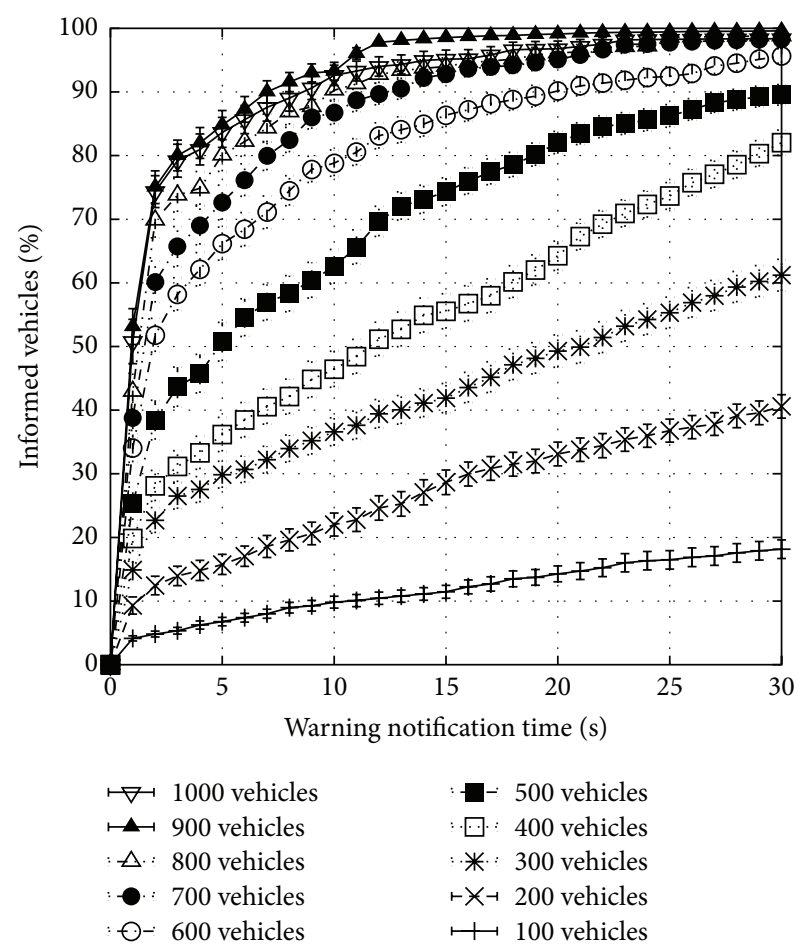

(b)

FIGURE 5: Warning notification time and percentage of vehicles informed when varying the number of vehicles in (a) San Francisco (USA) and (b) Valencia (Spain).

TABLE 5: Topology features of maps with similar TD.

\begin{tabular}{lcc}
\hline & SJR & TD \\
\hline Madrid & 0.8783 & 36.3 \\
Beijing & 1.2573 & 35.2 \\
\hline
\end{tabular}

the warning message dissemination process when varying the SJR and the TD, while maintaining the same number of vehicles per $\mathrm{km}^{2}$. The objective is to demonstrate that the communications performance strongly correlates not only with the number of vehicles, but also with the values of SJR and the TD of the roadmaps used (i.e., to the complexity and the capacity of them).

5.1. Similar TD and Different SJR Values. Table 5 shows the topology features of Madrid and Beijing. As shown, these cities present a similar Total Distance, whereas their SJR values are quite different. More specifically, although the capacity of vehicles is very similar in both maps, Madrid is a simple roadmap and Beijing is a complex one.

Figure 6 shows the percentage of vehicles informed in Madrid and Beijing when simulating 400 vehicles (i.e., $100 \mathrm{veh} / \mathrm{km}^{2}$ ). As expected, the percentage of vehicles informed is quite different. Madrid (a simpler map) results in an $84 \%$ of informed vehicles after the first 20 seconds, whereas the warning dissemination process in Beijing (a more complex map) is only able to achieve a $28 \%$ of vehicles informed along the same period of time. These results

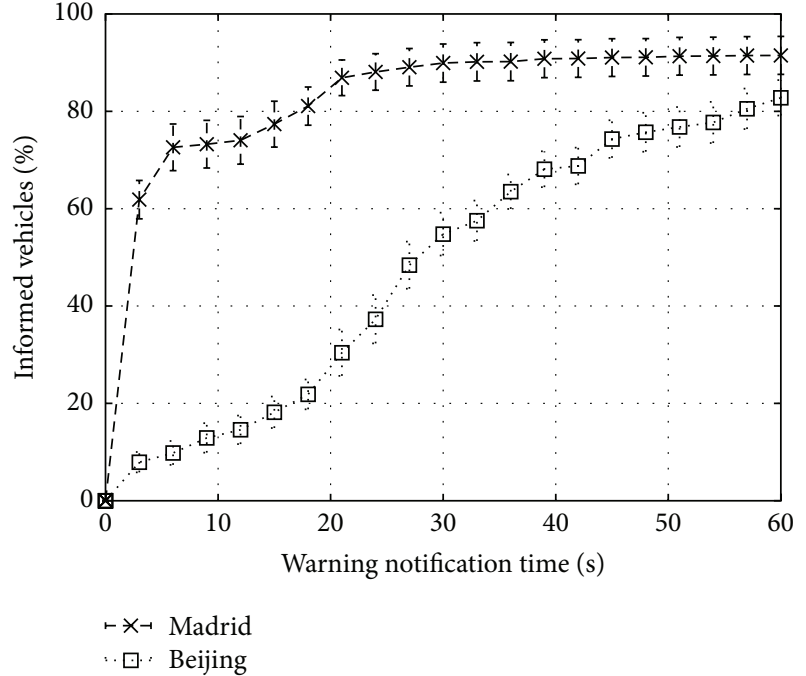

FIGURE 6: Percentage of informed vehicles in maps with similar TD and different SJR values.

confirm the importance of the SJ Ratio when disseminating warning messages, especially in urban environments. Although both maps present similar TD values, the results obtained in terms of vehicles informed are quite different.

5.2. Similar SJR and Different TD Values. Table 6 shows the SJ Ratio and the TD of Prague and Beijing. As shown, these cities 
TABLE 6: Topology features of maps with similar SJ Ratio.

\begin{tabular}{lcc}
\hline & SJR & TD \\
\hline Prague & 1.2419 & 62.1 \\
Beijing & 1.2573 & 35.2 \\
\hline
\end{tabular}

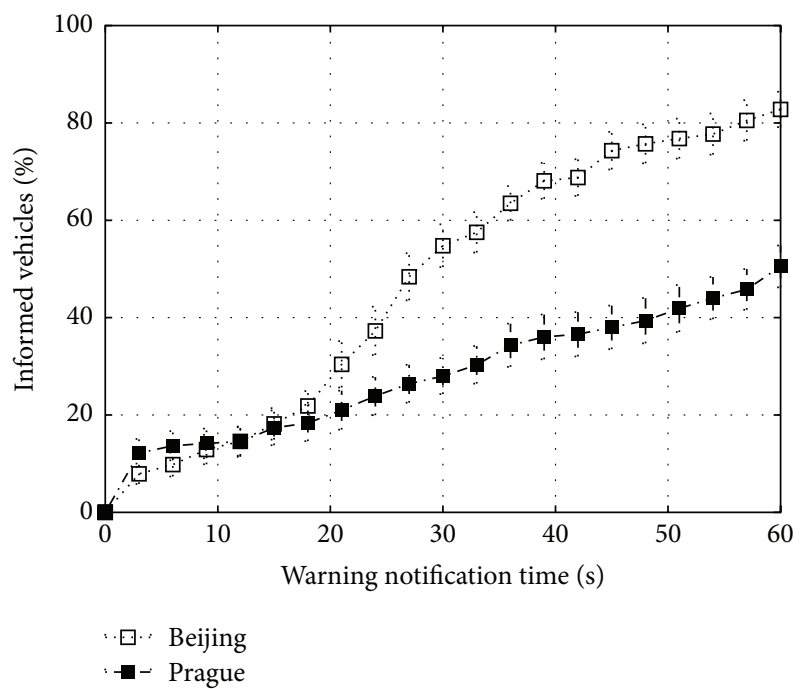

FIGURE 7: Percentage of informed vehicles in maps with similar SJR and different TD values.

present a very similar SJ Ratio, whereas their TD values are different. According to results presented in Section 5.1, both cities should result in similar communications performance since their complexity (in terms of SJR values) is quite similar. However, while Prague presents a TD of 62.1, Beijing hardly reaches 35.2 . As the TD is directly related to the maximum capacity of the roadmap (i.e., maps with higher TD values are able to host more vehicles), the number of vehicles per $\mathrm{km}^{2}$ should not be a fair vehicle density metric when comparing them. In fact, although the complexity of both maps is quite similar, the results obtained in terms of vehicles informed are different.

Figure 7 shows the results obtained in Prague and Beijing when simulating 400 vehicles. As shown, during the first 15 seconds the warning dissemination process behaves similarly since the complexity of both maps is quite similar in terms of SJ Ratio. However, the overall dissemination process is different since the higher TD of Prague makes the percentage of informed vehicles lower than in Beijing. Although the number of vehicles per $\mathrm{km}^{2}$ is the same, the probability of a successful communication is higher in Beijing than in Prague. These results confirm the importance of the TD metric when accounting for vehicular communications in different maps.

5.3. Similar TD and SJR Values. Finally, we focus on two different scenarios which present similar values for both parameters. Table 7 shows the SJ Ratio and the TD values of San Francisco and Madrid. As shown, both cities present very similar values. Hence, according to previous results,
TABLE 7: Topology features of maps with similar SJ Ratio and TD.

\begin{tabular}{lcc}
\hline & SJR & TD \\
\hline Madrid & 0.8783 & 36.3 \\
San Francisco & 0.8863 & 36.3 \\
\hline
\end{tabular}

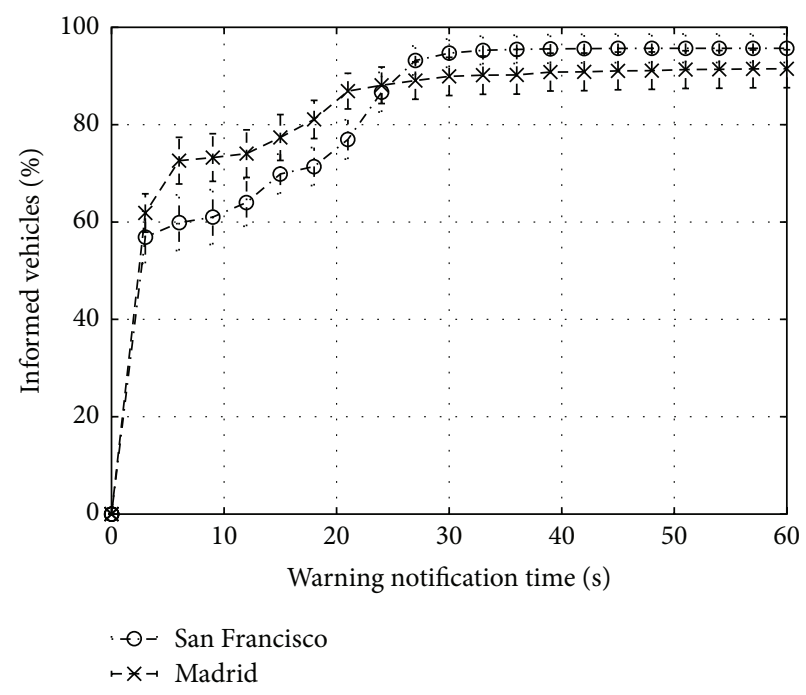

FIGURE 8: Percentage of informed vehicles in maps with similar SJR and similar TD values.

the communications performance in terms of vehicles informed in both cities should be very similar.

Figure 8 shows the results obtained under the same number of vehicles (i.e., when simulating 400 vehicles) in Madrid and San Francisco. In particular, results confirm that the communication process in maps which present similar values of both SJR and TD performs very similarly when the number of vehicles is also similar. Moreover, the percentage of informed vehicles remains quite similar during all the dissemination process.

\section{Novel Density Metric}

In the literature, we can find a plethora of VANET-based new applications, services, and protocols specially designed to propel future Intelligent Transportation Systems and mainly focused on increasing traffic safety and comfort of drivers and passengers. However, it is very common that authors only validate their proposals under very simplistic (and sometimes unrealistic) scenarios. In order to better validate their approaches, they should assess them under a different number of vehicles, realistic topologies, conditions, and so forth, thereby obtaining more significant conclusions.

According to the results presented in the previous section, we now propose a novel vehicle density metric which accounts not only for the number of vehicles and the area of the scenario but also for the complexity and the maximum capacity of the roadmap. Our metric will allow researchers to accurately characterize the different roadmaps and fairly validate their proposals. 
6.1. Our Proposal. In vehicular networks, it is very common to consider the vehicular density as the number of vehicles in a certain area. However, along this work, we have demonstrated that this metric is not suitable since the number of vehicles and the area of the scenario are not the only parameters highly affecting communication performance.

As expected, and similarly to mobility, communicating vehicles cannot reach all the vehicles located in the scenario. Therefore, and taking into account the special characteristics of the 802.11p standard, we consider that it is more accurate to account for the number of vehicles per kilometer of paved road including the number of lanes in each road. In addition, we have demonstrated that the layout of the roads also presents a noticeable influence in the communication capabilities, showing that scenarios with lower SJ Ratios ease communication among vehicles.

Taking into account these considerations, we propose a new metric to measure density in vehicular networks. Considering a scenario consisting of a map formed by a set $\mathbb{S}$ of streets, a set $\sqrt{ }$ of junctions, and a total area $_{\mathrm{km}^{2}}$, where a set $\mathbb{V}$ of vehicles are moving at a given time, we define density as shown in

$$
\begin{aligned}
& \operatorname{Density}(\mathbb{V}, \text { Map }) \\
& =\frac{\left(|\mathbb{V}| / \sum_{s \in \mathbb{S}} \text { length }(s) \cdot \text { num_lanes }(s)\right) \cdot(|\mathbb{I}| /|\mathbb{S}|)}{\text { Area }_{\mathrm{km}^{2}}} .
\end{aligned}
$$
in

We can further develop the previous equation as shown

$$
\begin{aligned}
& \operatorname{Density}(\mathbb{V}, \text { Map) } \\
& =\frac{\left(|\mathbb{V}| / \sum_{s \in \mathbb{S}} \text { length }(s) \cdot \text { num lanes }(s)\right) \cdot(|\mathbb{I}| /|\mathbb{S}|)}{\text { Area }_{\mathrm{km}^{2}}} \\
& =\frac{|\mathbb{V}|}{\sum_{s \in \mathbb{S}} \text { length }(s) \cdot \text { num_lanes }(s) / \text { Area }_{\mathrm{km}^{2}}} \cdot \frac{|\mathbb{J}|}{|\mathbb{S}|} \\
& =\frac{|\mathbb{V}|}{\operatorname{TD}(\text { Map })} \cdot \frac{1}{\operatorname{SJR}(\text { Map })} \\
& =\frac{\text { Number of vehicles }}{\mathrm{TD}(\text { Map }) \cdot \operatorname{SJR}(\text { Map })} \text {. }
\end{aligned}
$$

Note that unlike the traditional density metric which accounts for the number of vehicles per $\mathrm{km}^{2}$ our proposal also considers the complexity of the roadmap, and its maximum capacity; that is, our approach implicitly includes the communication possibilities among the vehicles. Therefore, our novel density metric should be used when accounting for vehicular communications in future Intelligent Transportation Systems. Note that vehicles can calculate the number of vehicles, the Total Distance, and the instant SJ Ratio in real-time since we consider that all the vehicles are equipped with communication devices and on-board GPS systems with integrated street maps.

The main objective is that researchers can characterize more precisely the different scenarios used in their simulations to better validate their proposals. Using our approach,
TABLE 8: Number of vehicles required to obtain similar density $(D)$ values.

\begin{tabular}{lccccc}
\hline & SJ Ratio & TD & $D=0.5$ & $D=1.25$ & $D=2.5$ \\
\hline Houston & 0.8430 & 43.6 & 18.4 & 46.0 & 92.0 \\
Madrid & 0.8783 & 36.3 & 16.0 & 39.9 & 79.8 \\
San Francisco & 0.8863 & 36.3 & 16.1 & 40.2 & 80.3 \\
Turin & 0.8958 & 43.0 & 19.3 & 48.2 & 96.3 \\
Los Angeles & 0.9379 & 32.3 & 15.1 & 37.9 & 75.7 \\
Amsterdam & 1.0310 & 37.1 & 19.1 & 47.8 & 95.5 \\
Sydney & 1.0712 & 34.2 & 18.3 & 45.9 & 91.7 \\
Liverpool & 1.1704 & 48.3 & 28.3 & 70.7 & 141.4 \\
Dubai & 1.2114 & 29.9 & 18.1 & 45.3 & 90.6 \\
Brussels & 1.2311 & 49.3 & 30.4 & 75.9 & 151.8 \\
Prague & 1.2419 & 62.1 & 38.6 & 96.4 & 192.9 \\
Beijing & 1.2573 & 35.2 & 22.1 & 55.3 & 110.5 \\
Valencia & 1.2678 & 47.8 & 30.3 & 75.7 & 151.4 \\
Rio de Janeiro & 1.3516 & 24.8 & 16.7 & 41.9 & 83.7 \\
Rome & 1.3881 & 32.8 & 22.8 & 57.0 & 114.0 \\
\hline
\end{tabular}

researchers simulating different roadmaps presenting the same density $(D)$ are guaranteed to obtain similar results in terms of communication performance.

6.2. Validation of the Novel Density Metric. In this subsection, we validate our proposed metric by studying the performance of the warning message dissemination process, in terms of warning notification time and percentage of informed vehicles. In particular, we simulate three different complexity scenarios (i.e., San Francisco, Amsterdam, and Valencia) under three different densities (i.e., low, medium, and high). Our objective is to demonstrate that our approach better correlates with the communication performance than the traditional density metric, allowing researchers to obtain similar results when simulating different roadmaps with a different number of vehicles.

Table 8 shows the number of vehicles required to obtain different densities (i.e., $D$ equal to $0.5,1.25$, and 2.5) for each scenario. For the sake of clarity, we also included the values of both SJR and TD parameters. According to this table, for example, if we simulate 76 vehicles in Los Angeles, 193 vehicles in Prague, and 114 vehicles in Rome, the results obtained in terms of vehicle communications should be very similar.

We now proceed to compare the communication performance when accounting for the traditional density approach in contrast to our proposed one. Specifically, Figures 9, 10, and 11 show the comparison in terms of percentage of informed nodes and warning notification time.

Figures 9(a) and 9(b) show the results when simulating a low density. More specifically, $100 \mathrm{veh} / \mathrm{km}^{2}$ (traditional metric) and $D=0.5$ (novel density metric), respectively. As shown, using the traditional approach, although the number of vehicles per $\mathrm{km}^{2}$ is the same, the percentage of informed vehicles is different for each of the scenarios simulated. However, despite the topology differences of the three maps, 


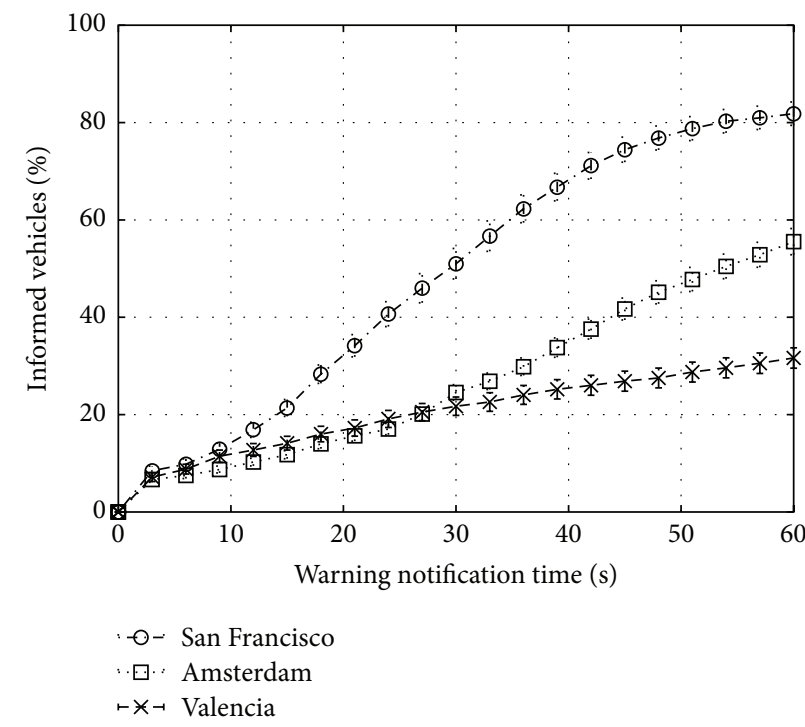

(a)

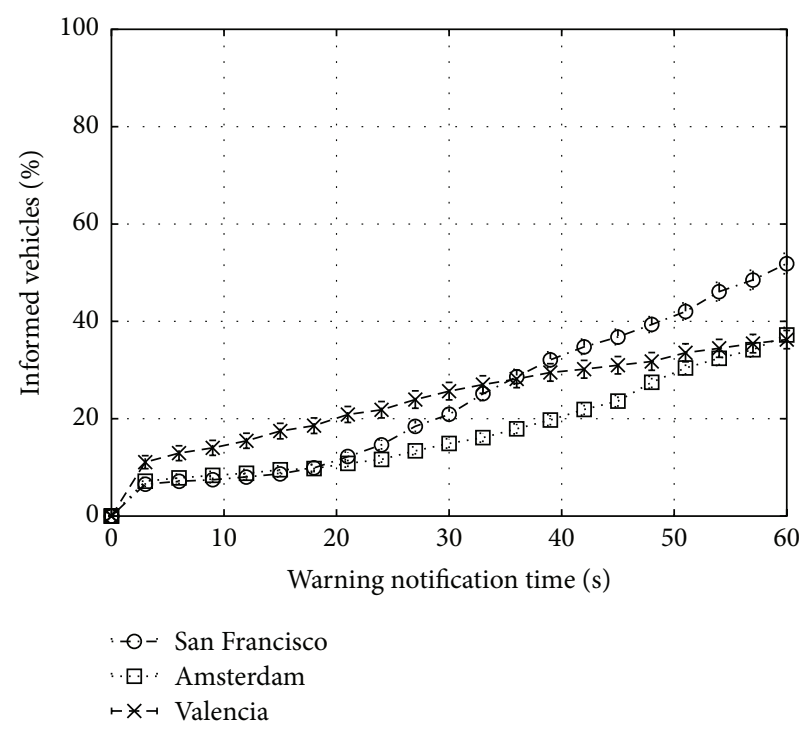

(b)

FIGURE 9: Comparison between the traditional density approach (a) and our proposed metric (b), under low-density conditions.

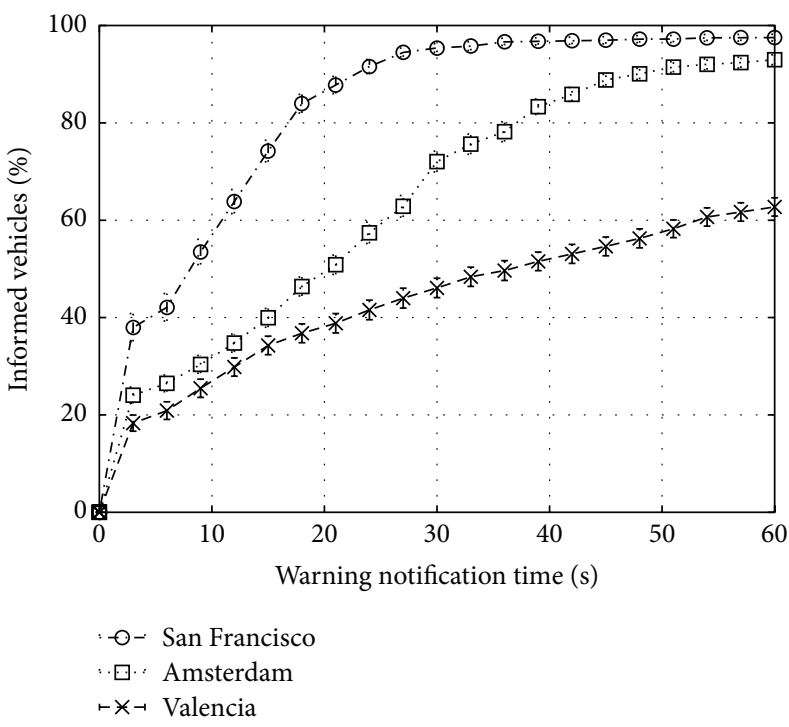

(a)

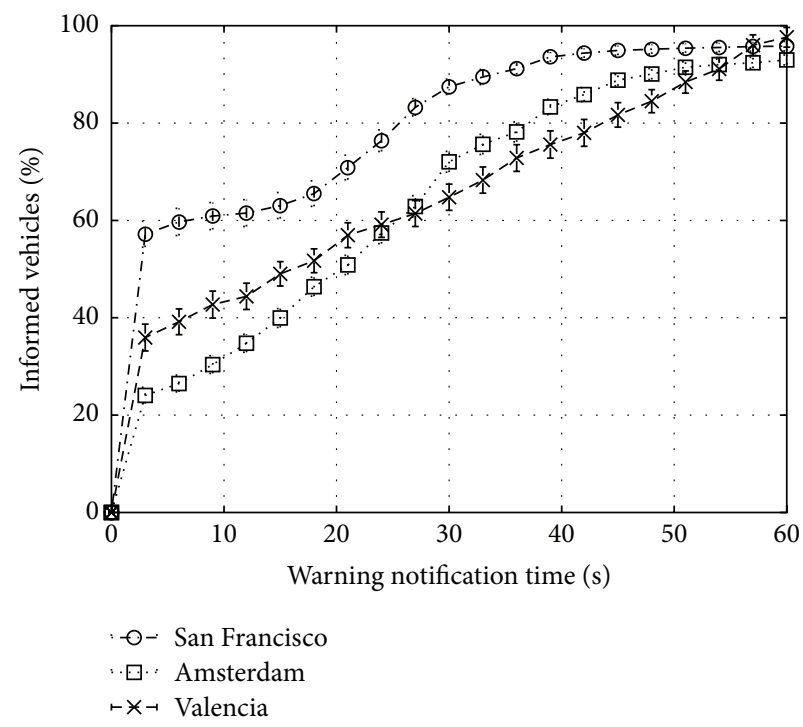

(b)

FIGURE 10: Comparison between the traditional density approach (a) and our proposed metric (b), under medium-density conditions.

the warning dissemination process behaves similarly during all the dissemination process, in terms of informed vehicles when using our density metric.

Figures 10(a) and 10(b) show the comparison under medium-density conditions (i.e., $200 \mathrm{veh} / \mathrm{km}^{2}$ and $D=1.25$, resp.). As shown, similarly to the previous results, communication performance is very similar for the different scenarios when using our density approach.

Finally, Figure 11 presents the results obtained under high-density conditions. In particular, we simulated $400 \mathrm{veh} /$ $\mathrm{km}^{2}$ (see Figure 11(a)) and $D=2.5$ (see Figure 11(b)). As shown, when accounting for the traditional density metric, Valencia results in lower percentage of informed vehicles, especially in the first part of the simulation (due to the higher complexity of the map), whereas when using our proposed density approach, the different roadmaps present similar values along all the simulated period.

\section{Conclusions}

Along this work we have analyzed the importance of the roadmap topology and the number of vehicles in vehicular networks performance. So far, only a few researchers have proposed classifications of different scenarios considering different parameters. In addition, we can find in literature 


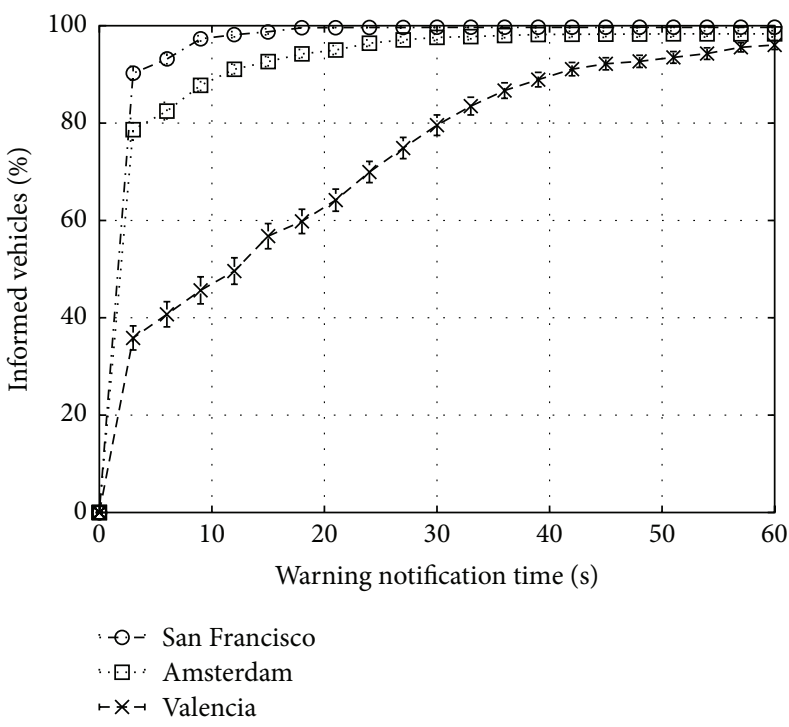

(a)

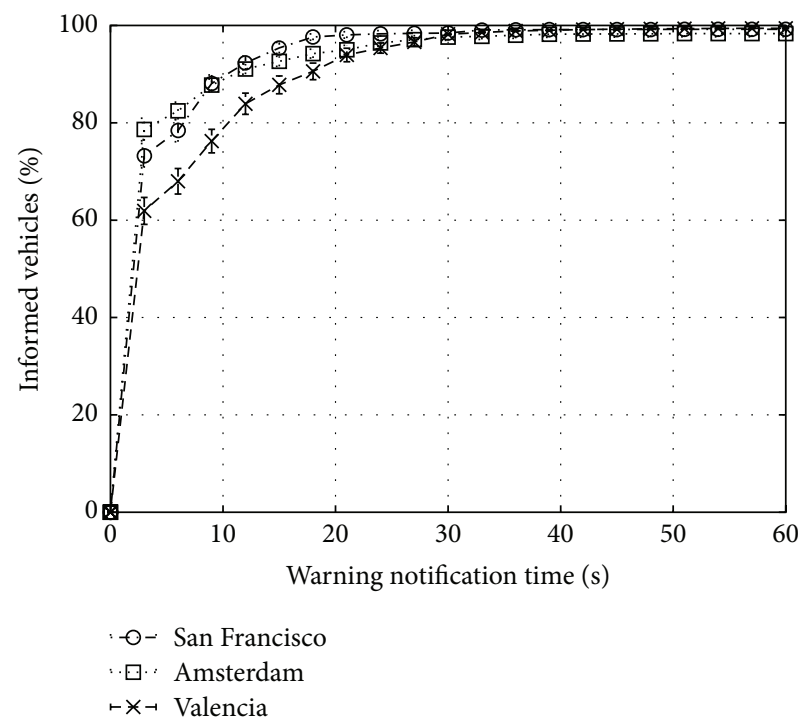

(b)

FIGURE 11: Comparison between the traditional density approach (a) and our proposed metric (b), under high-density conditions.

different criteria in order to measure the density in vehicular networks.

In order to help researchers to better characterize vehicular communications or assess their new VANET-based ITS proposals, we encourage them to account not only for the number of vehicles but also for the complexity of the roadmap and its maximum capacity. In particular, we propose the use of the SJ Ratio (SJR) and the Total Distance (TD) of maps to predict the communications possibilities of them. Simulation results confirm that not only the number of vehicles but also SJR and TD offer good correlation to communications performance. More specifically, maps with similar SJR and TD values offer similar results in terms of vehicles informed.

According to this, we propose a novel density metric which considers the number of vehicles, the complexity of the roadmap, and its maximum capacity. Our proposal allows researchers to accurately characterize the different urban scenarios and better validate their proposals related to cooperative Intelligent Transportation Systems.

\section{Conflict of Interests}

The authors declare that there is no conflict of interests regarding the publication of this paper.

\section{Acknowledgments}

This work was partially supported by the Ministerio de Economía y Competitividad, Programa Estatal de Investigación, Desarrollo e Innovación Orientada a los Retos de la Sociedad, Proyectos I D + I 2014, Spain, under Grant TEC2014-52690-R, and by the Government of Aragón and the European Social Fund (T91 Research Group).

\section{References}

[1] F. J. Martinez, C. K. Toh, J.-C. Cano, C. T. Calafate, and P. Manzoni, "Determining the representative factors affecting warning message dissemination in VANETs," Wireless Personal Communications, vol. 67, no. 2, pp. 295-314, 2012.

[2] Y. Khaled, M. Tsukada, J. Santa, J. H. Choi, and T. Ernst, "A usage oriented analysis of vehicular networks: from technologies to applications," Journal of Communications, vol. 4, no. 5, pp. 357368, 2009.

[3] J. Santa, R. Toledo-Moreo, M. A. Zamora-Izquierdo, B. Úbeda, and A. F. Gómez-Skarmeta, "An analysis of communication and navigation issues in collision avoidance support systems," Transportation Research Part C: Emerging Technologies, vol. 18, no. 3, pp. 351-366, 2010.

[4] Y. Liu, J. Ling, Q. Wu, and B. Qin, "Scalable privacy-enhanced traffic monitoring in vehicular ad hoc networks," Soft Computing, 2015.

[5] T. Delot, S. Ilarri, S. Lecomte, and N. Cenerario, "Sharing with caution: managing parking spaces in vehicular networks," Mobile Information Systems, vol. 9, no. 1, pp. 69-98, 2013.

[6] G. Mitra, C. Chowdhury, and S. Neogy, "Application of mobile agent in VANET for measuring environmental data," in Proceedings of the 1st International Conference on Applications and Innovations in Mobile Computing (AIMoC '14), pp. 48-53, IEEE, Kolkata, India, March 2014.

[7] G. Y. Cahng, J.-P. Sheu, and J.-H. Wu, "Typhoon: resource sharing protocol for metropolitan vehicular ad hoc networks," in Proceedings of the IEEE Wireless Communications and Networking Conference (WCNC '10), pp. 1-5, Sydney, Australia, April 2010.

[8] N. Qadri, M. Altaf, M. Fleury, and M. Ghanbari, "Robust video communication over an urban VANET," Mobile Information Systems, vol. 6, no. 3, pp. 259-280, 2010.

[9] P. Fazio, M. Tropea, F. Veltri, and S. Marano, "A new routing protocol for interference and path-length minimization in 
vehicular networks," in Proceedings of the IEEE 75th Vehicular Technology Conference (VTC Spring '12), pp. 1-5, IEEE, Yokohama, Japan, May 2012.

[10] Y.-C. Tseng, S.-Y. Ni, Y.-S. Chen, and J.-P. Sheu, “The broadcast storm problem in a mobile ad hoc network," Wireless Networks, vol. 8, no. 2-3, pp. 153-167, 2002.

[11] A. Alonso and C. Mecklenbraeuker, "Dependability of decentralized congestion control for varying VANET density," IEEE Transactions on Vehicular Technology, 2016.

[12] S. Das, R. Raw, I. Das, S. Sahana, and B. Purkayastha, "Effect of traffic density patterns on the performance of routing protocols for vanets," in Proceedings of the IEEE International Conference on Computing, Communication Automation (ICCCA '15), pp. 498-501, Noida, India, May 2015.

[13] A. T. Akabane, L. A. Villas, and E. R. Mauro Madeira, "An adaptive solution for data dissemination under diverse road traffic conditions in urban scenarios," in Proceedings of the IEEE Wireless Communications and Networking Conference (WCNC '15), pp. 1654-1659, New Orleans, La, USA, March 2015.

[14] J. A. Sanguesa, M. Fogue, P. Garrido, F. J. Martinez, J.-C. Cano, and C. T. Calafate, "Using topology and neighbor information to overcome adverse vehicle density conditions," Transportation Research Part C: Emerging Technologies, vol. 42, pp. 1-13, 2014.

[15] D. Jin, F. Shi, and J. Song, "A traffic flow theory based density adopted emergency message dissemination scheme for vehicular ad hoc networks," in Proceedings of the International Conference on Information Networking (ICOIN '15), pp. 57-62, Siem Reap, Cambodia, January 2015.

[16] J. Barrachina, P. Garrido, M. Fogue et al., "Reducing emergency services arrival time by using vehicular communications and Evolution Strategies," Expert Systems with Applications, vol. 41, no. 4, pp. 1206-1217, 2014.

[17] V. Tyagi, S. Kalyanaraman, and R. Krishnapuram, "Vehicular traffic density state estimation based on cumulative road acoustics," IEEE Transactions on Intelligent Transportation Systems, vol. 13, no. 3, pp. 1156-1166, 2012.

[18] E. Tan and J. Chen, "Vehicular traffic density estimation via statistical methods with automated state learning," in Proceedings of the IEEE Conference on Advanced Video and Signal Based Surveillance (AVSS '07), pp. 164-169, IEEE, London, UK, September 2007.

[19] N. Maslekar, M. Boussedjra, J. Mouzna, and H. Labiod, "A stable clustering algorithm for efficiency applications in VANETs," in Proceedings of the 7th International Wireless Communications and Mobile Computing Conference (IWCMC '11), pp. 1188-1193, IEEE, Istanbul, Turkey, July 2011.

[20] M. D. Venkata, M. M. M. Pai, R. M. Pai, and J. Mouzna, "Traffic monitoring and routing in VANETs-a cluster based approach," in Proceedings of the 11th International Conference on ITS Telecommunications (ITST '11), pp. 27-32, IEEE, Saint Petersburg, Russia, August 2011.

[21] J. A. Sanguesa, M. Fogue, P. Garrido et al., "An infrastructureless approach to estimate vehicular density in urban environments," Sensors, vol. 13, no. 2, pp. 2399-2418, 2013.

[22] K. Fall and K. Varadhan, "ns notes and documents," The VINT Project, UC Berkeley, LBL, USC/ISI, and Xerox PARC, 2000, http://www.isi.edu/nsnam/ns/ns-documentation.html.

[23] IEEE 802.11 Working Group, "IEEE Standard for Information Technology-telecommunications and information exchange between systems-local and metropolitan area networksspecific requirements-part 11: wireless LAN Medium Access
Control (MAC) and Physical Layer (PHY) specifications amendment 6: wireless access in vehicular environments," July 2010.

[24] F. J. Martinez, M. Fogue, C. K. Toh, J.-C. Cano, C. T. Calafate, and P. Manzoni, "Computer simulations of VANETs using realistic city topologies," Wireless Personal Communications, vol. 69, no. 2, pp. 639-663, 2013.

[25] M. Fogue, P. Garrido, F. J. Martinez, J.-C. Cano, C. T. Calafate, and P. Manzoni, "A realistic simulation framework for vehicular networks," in Proceedings of the 5th International Conference on Simulation Tools and Techniques (SIMUTools '12), pp. 37-46, Desenzano, Italy, March 2012.

[26] D. Krajzewicz, J. Erdmann, M. Behrisch, and L. Bieker, "Recent development and applications of SUMO-simulation of urban mobility," International Journal on Advances in Systems and Measurements, vol. 5, no. 3-4, pp. 128-138, 2012.

[27] OpenStreetMap, "Collaborative project to create a free editable map of the world," 2015, http://www.openstreetmap.org.

[28] European Telecommunications Standards Institute, "Intelligent Transport Systems (ITS); Communications Architecture. ETSI EN 302 665," 2010, https://www.etsi.org/deliver/etsi_en/302600 302699/302665/01.01.01_60/en_302665v010101p.pdf.

[29] S. Krauss, P. Wagner, and C. Gawron, "Metastable states in a microscopic model of traffic flow," Physical Review E, vol. 55, no. 5, pp. 5597-5602, 1997.

[30] J. A. Sanguesa, M. Fogue, P. Garrido et al., "RTAD: a realtime adaptive dissemination system for VANETs," Computer Communications, vol. 60, pp. 53-70, 2015.

[31] International Road Federation, IRF World Road Statistics (WRS), 2014, http://www.irfnet.ch/world_road_statistics.php.

[32] M. Artimy, "Local density estimation and dynamic transmission-range assignment in vehicular ad hoc networks," IEEE Transactions on Intelligent Transportation Systems, vol. 8, no. 3, pp. 400-412, 2007. 

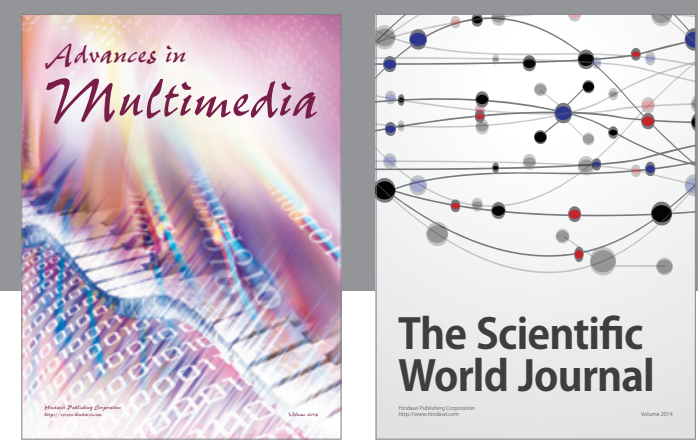

The Scientific World Journal
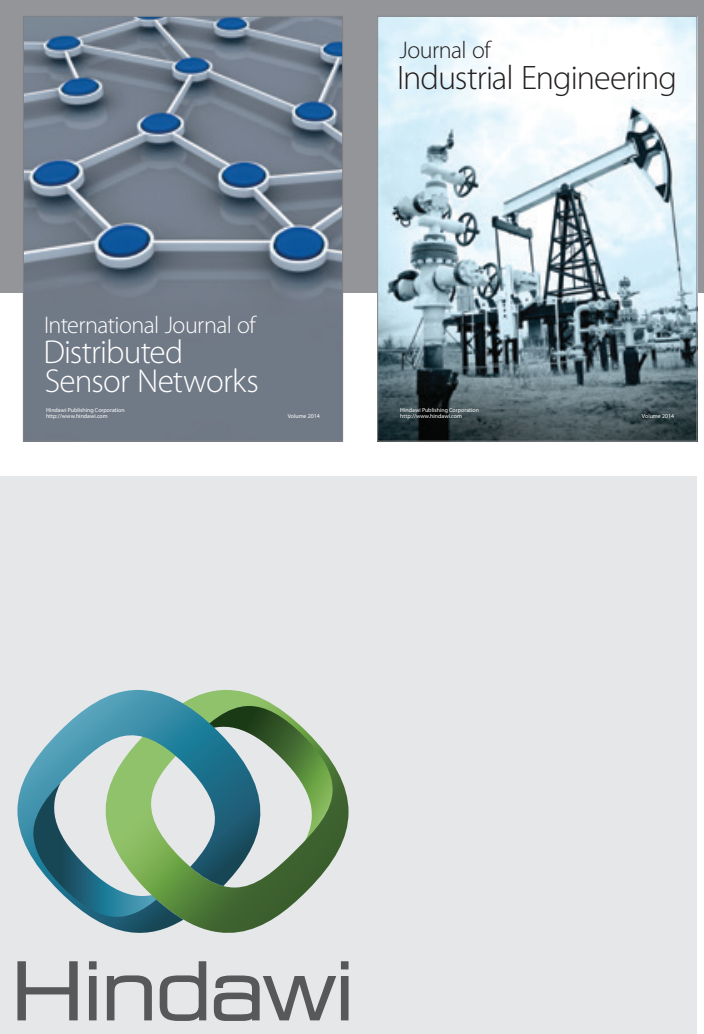

Submit your manuscripts at

http://www.hindawi.com

\section{Computer Networks} and Communications
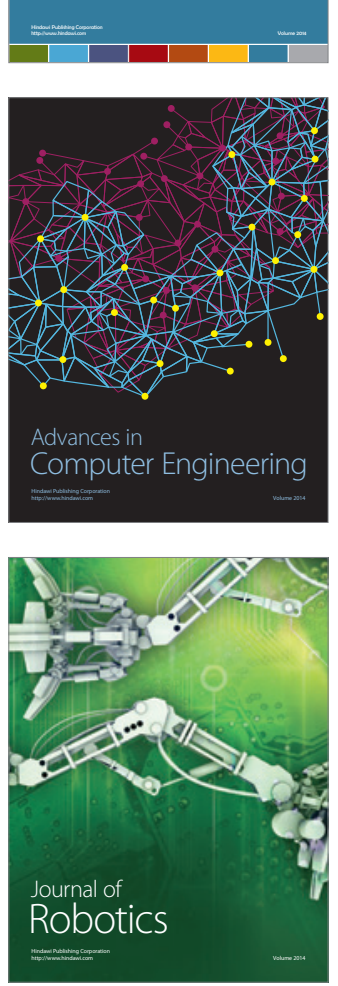
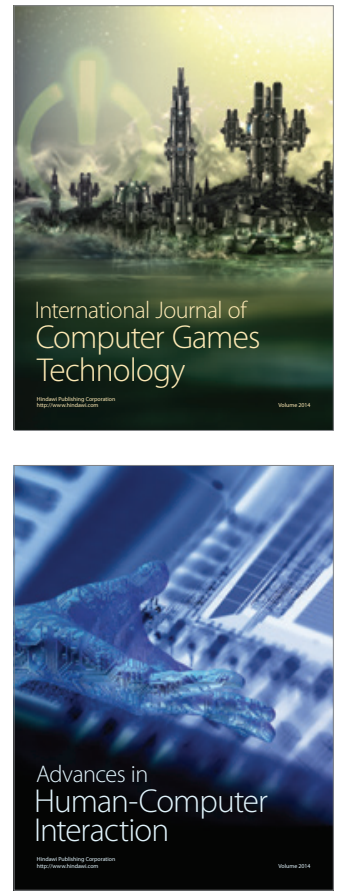
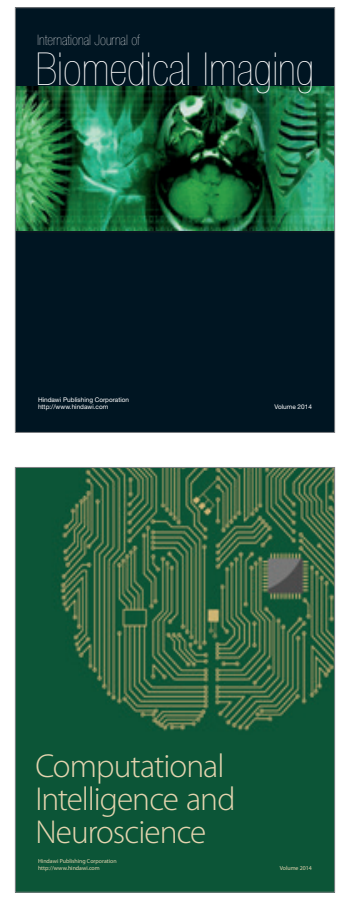
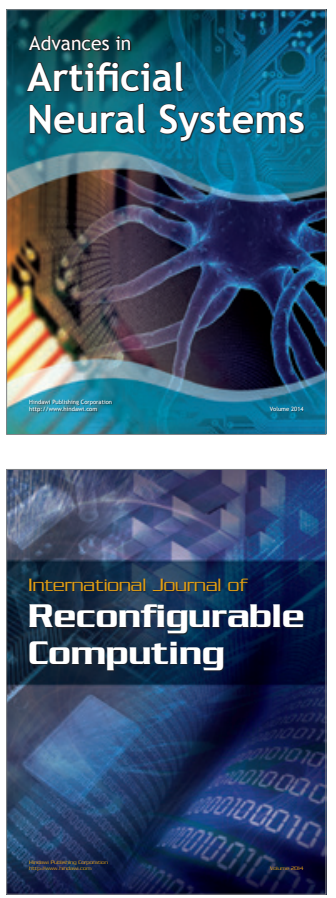
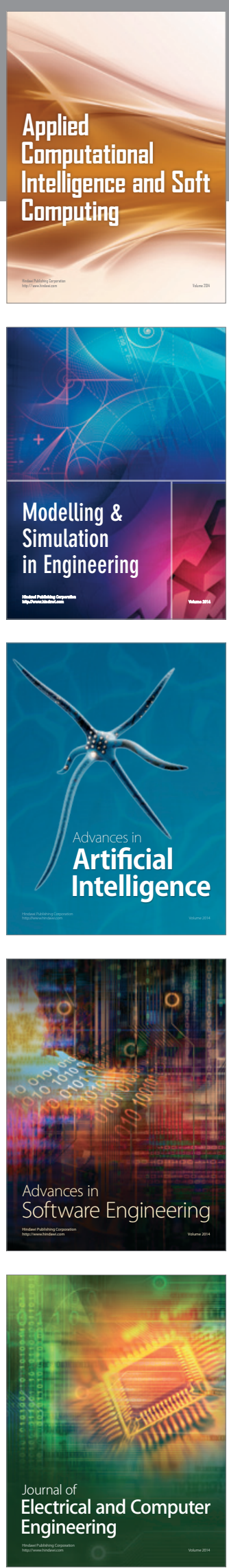